\title{
A Study on Forecaster Model using Time Series Data
}

\author{
Ashwini N. \\ Research Scholar, VTU Belagavi \\ Asst. Prof, Department of ISE \\ BMS Institute of Technology and Mgmt \\ Bengaluru, Karnataka, India
}

\author{
Rajshekar Patil M., PhD \\ Professor and Principal, \\ Department of CSE TCET (UD) \\ College of Engineering, Telangana State, India
}

\begin{abstract}
Many physical and artificial phenomena can be described by time series. The prediction of any such phenomenon could be complex and interesting. The ability to forecast the future is mainly based on only past data, which leads to strategic advantages and will be key to success in organizations. Time series forecasting allows the modeling of complex systems as black-boxes, being a focus of attention in several research arenas. There are several methods for time series data which mainly depends whether the data is linear or nonlinear. In this paper a survey on the forecasting method based on the different types of the data presented. This survey will mainly concentrate based on neural network, evolutionary computation etc. in solution development of forecasting models and rules, continued with hybrid forecaster mainly.
\end{abstract}

\section{Keywords}

Moving Average(MA), Autoregressive(AR), Neural Networks, Genetic algorithms, Time series forecasting, Autoregressive Integrated Moving Area(ARIMA) methods, Generalized autoregressive conditionally heteroskedastic(GARCH) methods, Artificial Neural Networks(ANN)

\section{INTRODUCTION}

Data mining is the process of finding previously unknown and potentially interesting patterns and relations in large databases [3]. Currently electronic data repositories are growing quickly and contain huge amount of data from commercial, scientific, and other domain areas. The capabilities for collecting and storing all kinds of data totally exceed the abilities to analyze, summarize, and extract knowledge from this data. Numerous data mining methods have recently been developed to extract knowledge from large databases. In many cases it is necessary to solve the problem of evaluation and selection of the most appropriate data-mining method or a group of the most appropriate methods. Often the method selection is done statically without analyzing each particular instance. If the method selection is done dynamically taking into account characteristics of each instance, then data mining usually gives better results.

Time series data is an ordered sequence of values of a variable at equally spaced time intervals [3]. In plain English, a time series is simply a sequence of data (usually numbers/images etc.) collected at regular intervals over a period of time.

One of the main characteristic of time series is that its stochastic nature. A stochastic process is a sequence of random variables, all defined on the same probability space with discrete time parameter (usually T). A stochastic process depends on both chance and time.

Time Series Analysis mainly deals with certain factors firstly data compression which provides compact description of the data. Secondly it deals with explanatory which are mainly a seasonal factors i.e. situation. deals with relationships with other variables (temperature, humidity, pollution, etc.). Other factor is signal processing which extracts a signal in the presence of noise. And finally prediction which uses the model to predict future values of the time series.

Time Series Analysis can be broadly divided into two categories depending on the type of the model used. The two categories are kinetic model and dynamic model. In kinetic model the data is used as a function of time i.e. $x t=f(t)$, observations of the process are seen as a function of time. Next type model is dynamic model where the data here is built-in as in equation (1).

$x t=f(x t-1, x t-2, x t-3, \ldots \ldots)$

The standard time series analysis techniques decomposes the time series function $x t=f(t)$ into up to four components [26]: Firstly is trend in a long-term monotonic change of the average level of the time series. Second component is the Trade Cycle which is a long wave in the time series. The third component is the Seasonal Component; it has fluctuations in time series that recur during specific time periods. Final component is the Residual component which represents all the influences on the time series that are not explained by the other three components. [27]

The time series prediction goal [3] is to observe or model the existing data series to enable future unknown data values to be forecasted accurately. Examples of data series include financial data series such as stocks, indices, rates, etc., physically observed data series like weather and mathematical data series like Fibonacci sequence, integral of differential equations, etc. The phrase Time series "generally refers to any data series, whether or not the data are dependent on a certain time increment.

To do time series prediction we can use many different forecasting techniques, which are based on historical time series data and the trends the data reveal. In modern research, different methods taken from a variety of fields are employed for this task.

During the prediction of time series a model has to be developed so we need to know time-series model, also called a signal model, which is a dynamic system that is identified to fit a given signal or time series data. The time series can be multivariate, which leads to multivariate models. A time series is modeled by assuming it to be the output of a system that takes a white noise signal $e(t)$ of variance Noise Variant(NV) as its virtual input. The true measured input size of such models is zero, and their governing equation takes the form $y(t)=H e(t)$, where $y(t)$ is the signal being modeled and $\mathrm{H}$ is the transfer function that represents the relationship between $\mathrm{y}(\mathrm{t})$ and $\mathrm{e}(\mathrm{t})$. The power spectrum of the time series 
is given by $*(N V * T s) * H^{\prime}$, where Ts is the model sample time.

The need for increased accuracies in time series forecasting has inspired the researchers to develop innovative models. In this paper, a survey is done to know how a forecaster model has the capability of exploiting the strengths of traditional time series approaches and artificial neural networks (ANNs).

Time series predation is one of the most important aspects for the practical usage of scientific and engineering knowledge, including physical science, daily temperature, control systems, engineering processes, bioengineering, environmental systems, business, management and econometrics. Real life problems and systems that adequately model such problems are often characterized by a large number of variables, parameters and interactions, resulting in highly complex non-linear dynamics, and in chaotic or random behavior. On the other hand, the amount of the available data collected to characterize the system is always limited. The problem of time series predation consists of predicting the next value of a series known up to a specific time, using the known past values of the series. Basically, time series prediction can be considered a modeling problem. The first step is establishing a mapping between I/O. Usually, the mapping is nonlinear and chaotic. After such a mapping is set up, future values are predicted based on past and current observations.

Construction of any data mining model follows in the Figure 1block diagram. Metadata consist information related to the model and the schema data on which the model works. As a result of mining patterns are obtained depending on the working of model. The model does contain a set of bindings, which point back to the data cached in the mining structure. If the data has been cached in the structure and has not been cleared after processing, these bindings enable you to drill through from the results to the cases that support the results. However, the actual data is stored in the structure cache, not in the model.

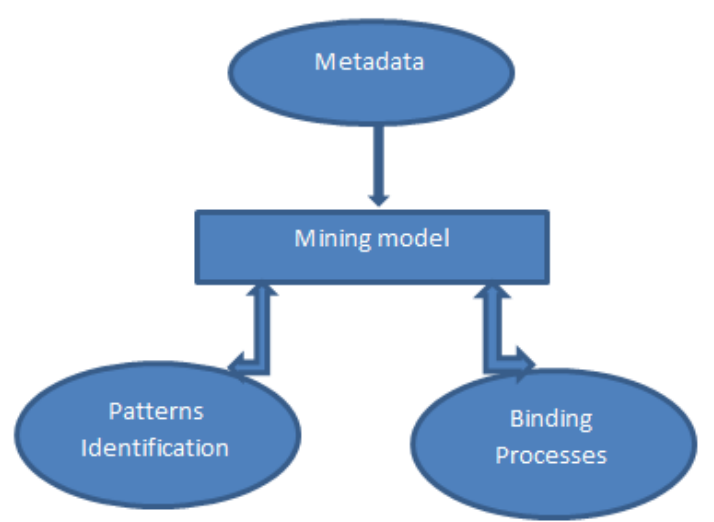

Figure 1: Mining Model

Time series forecasting has received tremendous attention of researchers in the last few decades. This is because the future values of a physical variable, which are measured in time at discrete or continuous basis, are needed in important planning, design and management activities. The time series forecasting methods have found various applications.

Time series forecasting (TSF), the forecast of a time ordered variable, turns on into a decisive tool in problem solving, since it allow one to model complex system where the goal is to predict the system's behavior and not how the system works. Indeed in the last few decades an increasing focus has been put over this field. Contributions from the arenas of operational research, statistics, and computer science as lead to solid TSF methods (e.g. Exponential smoothing or Regression) that replaced the old fashioned ones, where were primarily based on institution.

Mining the data in real time is very difficult since differentiating data as linear or nonlinear is a challenge. Hence we can develop a hybrid forecaster for forecasting both types of data with less forecasting errors.

Any system consists of different time series data which must be modeled. The study on the behavior of process and its metrics over an instant of period makes the basis for the time series model. During decision making, the uncertainty of the future makes time series models most effective for forecasting. Time series models generate certain anticipated results, using this anticipated results we can take future actions and decisions of that process. Anticipated results not only allow taking decisions but also used to improve forecasting techniques. Hence merging these models with other data mining practices we can recognize the comportment of the data and will be able to predict future drifts and patterns with real time data [27].

The models developed should be self-updating which includes predication of multiple time series. A self-updating model it is demanding forecasting model i.e. the requirements of the real world to be met. The requirements of the real world are the frequent changes i.e. new data to be added and irrelevant data to be removed from the forecasting data with respect to time ordered variable.

Behavior of time series is mainly dealt with the subsequent type's primarily ecological or environmental time series which has an increasing trend in linear, quadratic etc. Secondly is seasonal effect like monthly, quarterly, halfyearly, annually. Boundaries of some existing models are seasonal components which are static from year to year, stationary through the seasons and add intervention components like forest fires, volcanic eruptions, etc.

Hence an alternative approach for TSF [3] arises from that computational intelligence field, where one has observed a trend to look at nature for inspiration, when building problem solving models. In particular, studies on biological evolution influenced the loom of powerful artifacts, such as Genetic and evolutionary computation that enriched the potential use of intelligence in a broad set of scientific and engineering problem such as the ones of combinatorial and numeric optimization.

Evolutionary algorithms are suited for combinatorial optimization [3], where the exhaust of all possible solutions require enormous computational power, heuristically finding solution where other seems to fail. The use of ENN in TSF is expected to increase in importance, motivated by advantages such as explicit model representation and adaptive evolutionary search, which escape from unsatisfactory local minima.

\section{RELATED WORK ON FORECASTING METHODS}

Many real time organizations require data in different range, hence reference purposes we can include short ranged forecasts are defined herein as one to three years, medium 
range forecasts are defined as 3 to 5 years and long term forecasts are defined as greater than years. Greater than 10 years should be considered a historical data used for testing rather than a forecast. Several organizations like strategy groups delta with strategic planning are always in need for medium to long range forecasts. Sales and marketing groups demand medium range forecasts for resource planning. Business managers rely heavily on short and medium term forecasts for their own businesses data. Purchasing organization demands high quality forecasts for costs associated with raw materials, logistics, materials, as well as services.

Current time series forecasting methods generally fall into two groups: methods based on statistical concepts and computational intelligence techniques such as neural networks (NN) or genetic algorithms (GA). Hybrid methods combining more than one technique are also commonly found in the literature [9].

Naturally, the data collected over time consist of some random variation. There are few methods for dropping the effect of random variation in the data of a process. These methods are called smoothing methods. They are broadly classified into two: Averaging Methods and Exponential Smoothing Methods.

An average method is simple it is also called as mean method. In this methods it takes the average or mean of all the past data or observation for forecasting. This method is used for the when all the past observation are equally considered. This method is applied when there are no drifts. Average method can be mathematical written as where one $\mathrm{n}^{\text {th }}$ of the weight will be associated with each value of $\mathrm{x}$ as in equation (2).

$\bar{x}=\frac{1}{n} \sum_{i=1}^{n} x_{i}=\left(\frac{1}{n}\right) x_{1}+\left(\frac{1}{n}\right) x_{2}+\ldots+\left(\frac{1}{n}\right) x_{n_{n}}$

The other way of computing is by dividing the past data into smaller sets of taking the average of smaller sets. In this method we calculate on timely intervals $t 1, t 2, t 3 \ldots \ldots$. , the average value taken at $\mathrm{t} 1$ time slot will be dropped after calculating the average at $\mathrm{t} 2$. This type of averaging method is called moving average (MA) method mainly applied when there are drifts and it can be represented as follows where $\mathrm{X}$ is value

$M t=\frac{[x t+x t-1+\cdots+x t-N-1]}{N}$.

Statistical time series forecasting methods can be subdivided into the following categories: Exponential smoothing methods, Regression methods, Autoregressive integrated moving average methods, Threshold methods, Generalized autoregressive conditionally hetero-skedastic methods.

The first three categories as shown in Figure 2 can be considered a linear method that is methods that employ linear functional form for time series modeling and the last two are non-linear methods [10].

In exponential smoothing a forecast is given as a weighted moving average of recent time series observations. The values assigned decrease exponentially as the observations get older on time basis where as in average moving method all the type of values i.e. older or newer data is equally weighted. If we consider $y$ as the original observation of data, in exponential smoothing

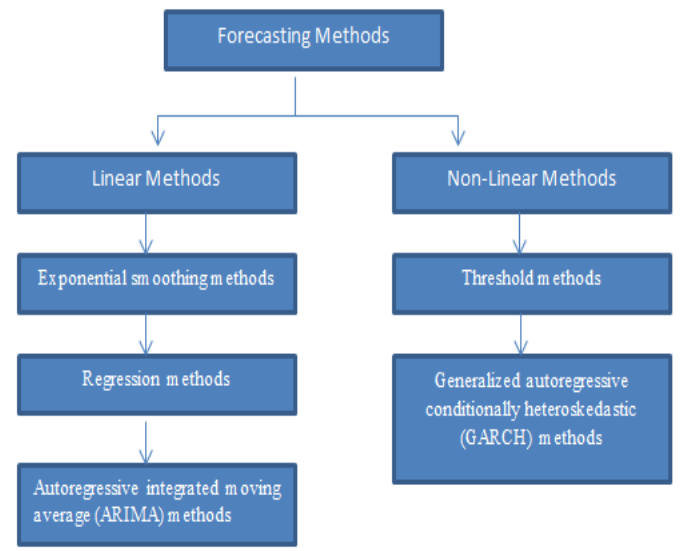

Figure 2: Classification of Forecasting Methods

method the smoothing scheme form second series $\left(\mathrm{S}_{2}\right)$ i.e. $\mathrm{S} 2=y$. For the third period, $S 3=\alpha y 2+(1-\alpha) S 2$; and so on. The time periods $1,2, \ldots \mathrm{n}$ are written as subscripts to $\mathrm{S}$. There is no S1; the smoothed series starts with the smoothed version of the second observation. For the time period $t$, the smoothed value $\mathrm{St}$ is found by computing the basic equation of exponential smoothing. The equation is as follows where $\alpha$ specifies the speed of older responses as in (4).

$S t=\alpha y t-1+(1-\alpha) S t-1,0<\alpha \leq 1, t \geq 3$.

Regression method of forecast is casual way of fitting the curves for the entire data set to minimize forecasting errors. It is given as a linear function of one or more explanatory variables. There are three types of regression linear, quadratic and cubic. The equations for the respective types are as in Table 1.

Table 1: Regression types with its equation

\begin{tabular}{|l|c|}
\hline Regression Type & Equation \\
\hline Linear & $X t=a+b t$ \\
\hline Quadratic & $X t=a+b t+c t 2$ \\
\hline Cubic & $X t=a+b t+c t 2+\mathrm{dt} 3$ \\
\hline Electing regression method: \\
\hline $\begin{array}{l}\text { As the higher order polynomial used then the degree of } \\
\text { errors can be reduced but the ability of forecasting also } \\
\text { reduces. Hence mainly we cubic approach. }\end{array}$ \\
\hline
\end{tabular}

Autoregressive Moving Average method uses both Autoregressive method and Moving Average method. Both $\mathrm{AR}$ and MA are linear regression methods but they vary in how they apply the current values of the time series. In AR method they apply current values on one or more prior values of the series where as in MA current value is applied on random shocks of one or more prior series. ARMA method can be represented with the following equation (5) where $X_{t}$ is the time series, $\mathrm{A}^{\mathrm{t}}$ is white noise, $\mu$ denotes is process mean.

$\mathrm{X}_{t}=\delta+\phi_{1} X_{t-1}+\phi_{2} X_{t-2}+\ldots+\phi_{p} X_{t-p}+$ $A_{t}-\theta_{1} A_{t-1}-\theta_{2} A_{t-2}-\ldots-\theta_{q} A_{t-q}$ 
ARMA method is mainly used for stationary process, in order to provide for non-stationary process we use ARIMA where 'I' stands for 'Integrated'. ARIMA [24] methods give a forecast as a linear function of past observations (or the differences of past observations) and error values of the time series itself and past observations of zero or more explanatory variables [3].

Threshold methods [3] assume that extant asymmetric cycles are caused by distinct underlying phases of the time series and that there is a transition period (either smooth or abrupt) between these stages. Commonly, the individual stages are given a linear functional form and the transition period (if smooth) is modeled as an exponential or logistic function. GARCH methods are used to deal with time series that display non-constant variance of residuals (error values). In these methods, the variance of error values is modeled as a quadratic function of past variance values and past error values.

Evolutionary algorithms [21] are suited for combinatorial optimization, where exhaust of all possible solutions require enormous computational power, heuristically finding solution where other seems to fail. Current time series forecasting methods generally fall into two groups: methods based on statistical concepts and computational intelligence techniques such as NN or GA. Hybrid methods combining more than one technique are also commonly found in the literature [26] [24].

Statistical time series forecasting methods can be subdivided into the following categories like Exponential smoothing methods, Regression methods, Auto Regressive Integrated Moving Average method are considered for linear methods that employ linear functional form for time series modeling. Threshold methods and Generalized Auto regressive conditionally heteroskedastic methods are considered as nonlinear methods [26].

In exponential smoothing a prediction is given as a weighted moving average of current time series annotations. The weights assigned decrease exponentially as the annotations get older. In regression, a prediction is given as a linear function of one or more explanatory variables. ARIMA methods gives a prediction as a linear function of past annotations or the differences of past annotations and it also considers error values of the time series itself and past annotations of zero or more explanatory variables [18]. Threshold methods [14] [11] assumes that present asymmetric cycles are caused by distinct essential phases of the time series and that there is a transition period which is either smooth or abrupt between these phases. Commonly, the distinct phases are given a linear functional form and the transition period if smooth is modeled as an exponential or logistic function. GARCH methods [12] [22] are used to deal with time series that can display non-constant difference of residuals (error values). In these methods, the difference of error values is modeled as a quadratic function of past difference values and past error values. The literature documenting statistical forecasting methods is vast. Many forecasting studies employ a variation on one of the techniques described above. Some studies [16] [19] employ statistical techniques to handle demand times series with infrequent characteristics.

Depending on the customized requirements methods are choice like regression trees [3][13] are used to handle forecasting demand for products influenced by promotions, Bayesian method [25] to forecast demand for products with very short demand histories. A system [6] can be dealt that uses statistical measures to help users manually select a forecasting model for a particular demand series.

Computational intelligence techniques for time series forecasting generally fall into two major categories: (i) Methods based on NN and (ii) Methods based on evolutionary computation.

Some recent NN forecasting studies includes recent literature on forecasting studies which make use of a hybrid model [15] that employs multiple methods. NN are commonly involved in hybrid models combining statistical and $\mathrm{NN}$ techniques [3][15][23]. Hybrid models will be in combination of genetic algorithms and neural networks or combining evolutionary programming and neural networks [4] or combining GP with an ARIMA model [5]. Some hybrid models use different techniques with genetic algorithm like statistical techniques [9] or diffusion modeling [7].

Real life problems and systems that adequately model such problems are often characterized by a large number of variables, parameters and interactions, resulting in highly complex non-linear dynamics, and in chaotic[14][8] or random behavior due to which the amount of the available data collected to characterize the system is always limited.

\section{FORECASTING MODELS APPLICATIONS}

For security application a time series hybrid model was developed by Samuel H. Huddleston and Donald E. Brown [4]. This model was used to check the effectiveness of predicting system to support routine prediction of criminal events in security applications. Some of the criminal events to be forecasted include resources, assigning, and conducting unit performance assessment. Here they have used the most popular geographical probability forecasting with HoltWinters exponential smoothing and ARIMA method for crime detection. This method is an hybrid model which is successful for only linear data.A simulation model is been developed which gives a comparative statement for forecasting methods for criminal events in security applications. Using Mean Absolute Scaled Error (MASE) method a comparative analysis, for which forecasting methods gives best performance for respective parameters. Comparative statement is with the following methods GPF-HW, GPFARIMA, naive, ARIMA.

For decomposing forecast data into linear and non-linear Mehdi Khashei and Mehdi Bijari [5] proposed a novel hybrid model which is developed. In the previous hybrid model, is on linear data hence will be more successful. The problem will occur only when the data is in the combination of linear and non-linear data. In a paper a novel hybridization model has been developed using ARIMA and ANN models. ARIMA model is used to recognize and extend the existing linear structure in time series data. ANN model is used to determine a model to capture the underlying data generating process and forecast using preprocessed data.

For improvising forecasting performance Yi-Shian Lee and Lee-Ing Yong has discussed an hybrid model with ARIMA method and Genetic Programming (GP) method [9]. As ARIMA method is highly accurate for linear problems not for non-linear problems. Hence a hybrid forecaster developed for 
non-linear problems which include ARIMA and GP methods. They have used GP parse tree representation and Support Vector Machine (SVM) model to validate the effectiveness of the proposed predicting model. The above model is tested with different data sets like Energy consumption, Finances of quarterly / Half-yearly report. Here the complete data set is given the segregation of data between linear and non-linear is done by the hybrid forecaster. Comparisons with graphs are done in order to check the improvising forecasting performance.

For disaggregation and aggregation of time series data a hybrid forecaster is developed by Theodosiou M [7]. This hybrid forecaster consists of generalized regression neural networks and Theta method. Generalized regression is used for out of sample exploration. They have concentrated on strengths and weakness of generalized regression neural networks. STL decomposition is used for decomposing constitute components. To each such component generalized regression is applied. Then apply theta method for further forecasting with respect to components.

Time series analysis places a vital role in medical science. A disease out breaking system [2] was developed for analyzing how may will be infected for an interval time. This system was developed by Richard John M Buendia uses ARMA model. They had used ARIMA method for short term forecasting and on a longer run ARMA model was supporting. ARMA method was developed for two context one for understanding the background of data source and the other is to make predictions.

As many real world scientific and engineering problems are irregular, enumerative and deterministic search techniques [1][3] are then unsuitable. Stochastic search and optimization approaches were developed as alternative approaches for solving these irregular problems. Stochastic methods require a function assigning fitness values to possible (or partial) solutions, and encode /decode (mapping) mechanism between the problem and algorithm domains. In general stochastic methods provide good solutions to a wide range of optimization problems which traditional deterministic search methods find difficult.

\section{PROPOSED SOLUTION}

Neural networks have been used in applications of prediction for time series. Number of different neural networks and learning methods has been applied to the times series prediction problem with varying degrees of success .The multi-layer perceptron's is probably the most frequently used type of neural networks [3]. However, due to its multi-layered structure and the greedy nature of the back propagation training algorithm, the training processes using BackPropagation may produce problems in training, as converge to local minima of the error surface or converge too slowly so as to affect the reliability and accuracy of prediction model With the intelligent integration of different architecture and evolution, ANNs can be able to approximate data with nonlinear and non-stationary properties and to conduct selflearning adaptive structure identification and parameter estimation in modeling process.

Many physical and artificial phenomena can be described by time series. The prediction of such phenomenon could be as complex as interesting. There are many time series forecasting methods, but most of them only look for general rules to predict the whole series. The main problem is that time series usually have local behaviors that don't allow forecasting the time series by general rules. In this research, focus will be given for finding a new local prediction rules. Those local prediction rules can attain better general prediction accuracy. The method in this research will be based on the evolution of a rule system.

\section{OBJECTIVES OF TIME SERIES}

Chaotic behavior in deterministic dynamical systems is an intrinsically non-linear phenomenon [3]. A characteristic feature of chaotic systems is an extreme sensitivity to changes in initial conditions, whiles the dynamics at least for so-called dissipative systems, is still constrained to a finite region of state space called a strange attractor. Time traces of the state variables of such systems display a seemingly stochastic behavior. In this research, concept of evolution will applied to evolve neural network at various level for predicting the time series with higher accuracy. Various benchmarks of chaotic time series like Logistic differential equation, Micky-glass and Lorenz system will take for experiment along with some other time series data set.

Also if we are able to fix the following objectives then any type of real time problems can be fixed that is design a forecasting model for chaotic system, develop evolutionary learning algorithms or rules for forecasting models. Then design a hybrid forecaster to improve forecasting performances. And finally a ccomparative performance analysis can be done with obtained results of existing models or results in literatures.

\section{CONCLUSION}

Time series analysis and forecasting is an active research area over the last few decades. The accuracy of time series forecasting is fundamental to many decision processes and hence the research for improving the effectiveness of forecasting models has never stopped. By designing a hybrid forecaster with neural networks and evolutionary computation with generalised rules for prediction irrespective to the real time data and by performance improvising we can get an accurate forecaster.

\section{REFERENCES}

[1] Zhenfeng Shao, Yuan Zhang and Weiqi Zhou, "Longterm monitoring of the urban impervious surface mapping using time series Landsat imagery: A 23-year case study of the city of Wuhan in China" 2016 4th International Workshop on Earth Observation and Remote Sensing Applications (EORSA), Guangzhou, 2016:212-216. (doi:10.1109/EORSA.2016.7552799)

[2] Richard John M Buendia and Geoffrey A Solano, "A Disease Outbreak Detection System using Autoregressive Moving Average in Time Series Analysis", published in 6th International Conference on Information, Intelligence, Systems and Applications(IISA),Corfu,2015;1-5. (doi: 10.1109/IISA.2015.7388087.)

[3] Ashwini N and Bhavya G, "Using Mining and Intelligent Approach for Time Series Forecasting Problems" ,International Journal of Advanced Research in Computer Science and Software Engineering, November 2013;3(11);1567-1571.

[4] Samuel H. Huddleston and Donald E. Brown,"Using Discrete Event Simulation To Evaluate Time Series 
Forecasting Methods For Security Applications", Proceedings of the Winter Simulation Conference, 2013;2772-2783.

[5] Mehdi $\mathrm{K}$ and Mehdi B, "A novel hybridization of artificial neural networks and ARIMA models for time series forecasting”, Applied Soft Computing, 2011;11.

[6] Lee $\mathrm{Y}$ and Tong L, "Forecasting time series using a metho3dology based on autoregressive integrated moving average and genetic programming", KnowledgeBased Systems, 2011; 24; 66-72.

[7] Theodosiou M, "Disaggregation and aggregation of time series components: a hybrid forecasting approach using generalized regression neural networks and the theta method", Neurocomputing,2011;20;896-905.

[8] Azadeh, A. and Faiz, Z. , "A meta-heuristic framework for forecasting household electricity consumption", Applied Soft Computing, 2011;11; 614-20.

[9] Lee Y and Tong L, "Forecasting time series using a methodology based on autoregressive integrated moving average and genetic programming", Knowledge-Based Systems, 2011;24; 66-72.

[10] Araujo. R., "A quantum-inspired evolutionary hybrid intelligent approach for stock market prediction", International Journal of Intelligent Computing and Cybernetics, 2010;3; 24-54.

[11] Chen, S. and Chen, J., "Forecasting container throughputs at ports using genetic programming", Expert Systems with Applications, 2010;37;2054-8.

[12] Chern, C., Ao, I., Wu, L. and Kung, L., "Designing a decision-support system for new product sales forecasting", Expert Systems with Applications, 2010; 37; 1654-65.

[13] Dilip, P. , "Improved forecasting of time series data of real system using genetic programming", GECCO '10 Proceedings of the 12th Annual Conference on Genetic and Evolutionary Computation, Portland, OR, USA, 2010;1;977-8.

[14] Chen, A. and Leung, M., "A Bayesian vector error correction model for forecasting exchange rates", Computers \& Operations Research, 2003;30;887-900.

[15] McMillan, D.G. , "Nonlinear predictability of stock market returns: evidence from nonparametric and threshold models", International Review of Economics and Finance, 2001;10;353-68;

[16] Chiraphadhanakul, S., Dangprasert, P. and Avatchanakorn, V., "Genetic algorithms in forecasting commercial banks deposit", Proceedings of the IEEE International Conference on Intelligent Processing Systems, Beijing, China, 1997;1;557-65.

[17] Back, T., "Evolutionary Algorithms in Theory and Practice: $\quad$ Evolution Strategies,Evolutionary Programming, and Genetic Algorithms", Oxford University Press,New York, NY, 1996.

[18] Chambers, L. (Ed.), "Practical Handbook of Genetic Algorithms: Applications", CRC Press,Boca Raton, FL, 1995.

[19] Clements, M. and Hendry, D. , "Forecasting in cointegrated systems", Journal of Applied Econometrics, 1995;10;127-46.

[20] Fogel, L., Angeline, P. and Fogel, D. , “An evolutionary programming approach to self-adaptation on finite state machines", Proceedings of the 4th Annual Conference onEvolutionary Programming, San Diego, CA, USA,1995;1; 355-65.

[21] Baille, R. and Bollerslev, T. , "Cointegration, fractional cointegration, and exchange rate dynamics", Journal of Finance, 1994;49;737-45.

[22] Cheung, Y. and Lai, K., "A fractional cointegration analysis of purchasing power parity", Journal of Business and Economic Statistics, 1993;11;103-12.

[23] Akgiray, V., "Conditional heteroskedasticity in time series and stock returns: evidence and forecasts", Journal of Business, 1989;62;55-80.

[24] Bollerslev, T. , "Generalized autoregressive conditional heteroskedasticity", Journal of Econometrics, 1986;31; 307-27.

[25] Engle, R., "Autoregressive conditional heteroskedasticity with estimates of the variance of UK inflation", Econometrica, 1982;50;987-1008.

[26] Bass, F. , "A new product growth model for consumer durables", Management Science,1969;15;215-27.

[27] https://www.google.co.in/?gfe_rd=cr\&ei=AMqKWNvyE ceL8Qewr7zIAg\#q=survey+on+Statistical+time+series+ forecasting+methods 